\title{
Herramientas de estrategias Web 2.0 para el desarrollo de la inteligencia emocional en estudiantes universitarios
}

\author{
Web 2.0 strategy tools for the development of emotional intelligence in \\ university students
}

\section{LUISA SERRA}

serra_sl@yahoo.com

Código ORCID: 0000-0002-7561-2541
- MARLE MARTÍNEZ

marlemartinez@yahoo.es

Código ORCID: 0000-0002-1531-3320
- JOSEABEL CEGARRA

joseabelcegarra@gmail.com

Código ORCID: 0000-0001-5395-528X

Universidad del Zulia, Departamento de Ciencias Formales, Núcleo LUZ-COL

Resumen La investigación tuvo como propósito especificar los tipos de herramientas de las estrategias Web 2.0 para el desarrollo de la inteligencia emocional en estudiantes, de la Licenciatura en Educación Mención Informática del Núcleo Costa Oriental del Lago de la Universidad del Zulia. El estudio consistió en una investigación acción, que recupera la reflexión crítica sobre la práctica docente. Posee un paradigma positivista, el tipo de investigación fue proyectiva con nivel descriptivo, diseño de campo, no experimental y transversal. Se diseñó un cuestionario, constituido por 18 ítems de tipo dicotómico. Así mismo, se estimó la confiabilidad utilizando el coeficiente Alfa de Cronbach, resultando en 0.9. Se concluyó que las estrategias Web 2.0, pueden desarrollar la inteligencia emocional, en aspectos como el control, expresión adecuada de emociones, liderazgo, afectividad, empatía y toma de decisiones.

Palabras clave: Estudiantes universitario; Herramientas Web 2.0; inteligencia emocional; Internet

\begin{abstract}
The purpose of the research was to specify the types of tools of the Web 2.0 strategies for the development of emotional intelligence in students, from the Bachelor's degree of Education Mention Informatics at the University of Zulia on the Eastern coast of Maracaibo. The research consisted of an action research approach, which recovers the critical reflection on teaching practice. It has a positivist paradigm, the type of research was projective with descriptive level, field design, non-experimental and transversal. A questionnaire was designed, consisting of 18 dichotomous items. Likewise, reliability was estimated using Cronbach's Alpha Coefficient, resulting in 0.9. It was concluded that Web 2.0 strategies can develop emotional intelligence, in aspects such as control, adequate expression of emotions, leadership, assertiveness, empathy and decision making.
\end{abstract}

Keywords: Web 2.0 Tools; emotional intelligence; university students 


\section{INTRODUCCIÓN}

Algo que no cambia en las aulas de clases es el primer encuentro del docente con los alumnos, este es un momento motivador, muchas veces define conductas, criterios, hasta vidas, es definitivo que de allí dependen muchas cosas, una de ella es la inspiración por aprender. Tomando lo anterior como punto de partida, fusionar estrategias Web 2.0 con el desarrollo de la inteligencia emocional, es un tema de gran relevancia actualmente, con el transcurrir del tiempo, se han realizado diversos estudios sobre lo referente a la Inteligencia Emocional (IE) y su influencia sobre el recurso humano, la cual parte de la existencia del referido factor, tal como lo afirman Goleman (2013) y Acosta (2013) en sus diferentes planteamientos.

En este orden de ideas, sin duda vale mucho la interacción amena en el aula de clase, el clima de aprendizaje debe ser propicio, en él se crea el amor a los estudios, la lectura, los libros, el amor a un arte, ciencia o práctica, aprender de errores (como cuando se es niño) es una experiencia reparadora que debe ser entendida e igualmente concebida de forma positiva.

La Web 2.0 también ha revolucionado la forma en que se distribuye el material escrito, en consecuencia la información, el conocimiento, ahora es posible conseguir gran parte del material escrito a través de medios digitales en forma de archivos digitales, la difusión de las noticias más importantes, ahora se da a través de páginas Web de los propios diarios, e incluso han surgido diarios cuya existencia sólo se desarrolla en la Web.
En el caso venezolano, actualmente los elementos educativos basados en la Web son bastante difundidos, aunque se lucha con las dificultades técnicas de la poca o nula conectividad a Internet, los escasos medios tecnológicos dentro de los institutos educativos y la resistencia a los paradigmas que rompen con la comodidad de lo tradicional, sin embargo, aun con todos esos factores en contra la educación se abre espacios para el desarrollo; por tal motivo se contextualiza el presente estudio dentro de una las instituciones educativas a nivel superior más reconocido dentro del contexto educativo.

A través de un diagnóstico preliminar, realizado mediante la observación directa y entrevistas no estructurada a un grupo de estudiantes de la Universidad del Zulia, Núcleo Costa Oriental del Lago, se detectaron ciertos problemas vinculados con el manejo emocional, cuando se utilizan las herramientas Web 2.0. En ese sentido, los mismos pudieran ser gobernados por sus emociones y utilizan las redes sociales como vías de escape, en lugar de orientar su uso para la construcción de conocimiento, mejorar las relaciones interpersonales y alinear esfuerzos para el logro de una aprendizaje significativo mediante la colaboración.

Con base en lo anteriormente expuesto, el problema está vinculado con el hecho que los estudiantes, pueden verse afectados por emociones distintas al momento de aprender sistemas operativos.

Por otra parte, si persiste la situación, se prevé el surgimiento de problemas personales en las relaciones entre los estudiantes, docentes e incluso entorno general, ya que se 
ha evidenciado anteriormente que el hombre es un ente biopsicosocial, por lo cual, es necesario emprender investigaciones que traten de estudiar la inteligencia emocional y la Web 2.0. Para ello, se requiere Determinar el uso de las estrategias Web 2.0 para el desarrollo de la inteligencia emocional en los estudiantes de la unidad curricular sistemas operativos, de la Licenciatura en Educación Mención Informática del Núcleo LUZ COL.

A partir de lo anterior surge la idea de implementar estrategias usando herramientas de la Web 2.0 en los estudiantes de la Unidad Curricular Sistemas Operativos, de la Licenciatura en Educación Mención Informática del Núcleo LUZ COL. Con el principal objetivo que los estudiantes, quienes cursan la unidad curricular, adquieran los conocimientos y competencias tecnológicas mediante el uso de las herramientas de la Web 2.0.

En el marco de la realización de esta investigación se estimaron un conjunto de referentes que permitieron ampliar la visión crítica en torno al objeto de estudio. Al respecto, se destacó que los estudiantes deben aprender a aprender con un enfoque profundo y no superficial, para ello es muy importante la orientación de los profesores a fin de adquirir hábitos de estudio adecuados y desarrollen estrategias de aprendizaje que puedan utilizar de acuerdo con la meta u objetivo a alcanzar, lo importante es aplicar los recursos y las herramientas en cualquier momento o contexto de aprendizaje.

Cuando se habla de estrategias aplicadas como parte de un ambiente de aprendizaje, se hace referencia a aquella secuencia ordenada y sistematizada de actividades y recursos que los docentes emplean en la práctica educativa, para ayudar el aprendizaje de los estudiantes, con clara intencionalidad pedagógica. El uso de la Web para la difusión de información y conocimiento desde la perspectiva escolar ha derivado en diferentes corrientes, desde el aprendizaje en línea a través de la búsqueda de información de manera autónoma y casi autodidacta.

La Web ha evolucionado desde las clásicas páginas de sólo lectura que constituían una simple vitrina de contenidos, hacia un conjunto de tecnologías y herramientas que la convirtieron en una plataforma abierta basada en la participación de los usuarios, adquiriendo así una nueva dimensión social y participativa. Los usuarios ahora pueden también ser creadores de contenidos, logrando interactuar con otros usuarios, dándole esto un nuevo sentido a la Web, dejando de ser un elemento de consumo y transformándose en un elemento de producción y creación de los usuarios.

En este orden de ideas, la Web 2.0, en el paradigma actual, se acerca al ideal de Internet entendida como escenario de interacción social, capaz de dar soporte tecnológico al desarrollo de una comunidad virtual, recurso activo de una auténtica sociedad de la información y del conocimiento. Además, las compañías que habían sobrevivido al colapso parecían tener algunas cosas en común. ¿Podría ser que el colapso marcó algún tipo de punto de inflexión para la web, de modo que una llamada a la acción como "Web 2.0" podría tener sentido? de acuerdo en que así fue, y resultando así la primera Conferencia Web 2.0. 
Por ello, la experiencia de interacción del usuario como fortaleza del entorno Web 2.0. Lejos ha quedado la preferencia del usuario por las páginas recargadas de artificios animados que ofrecían los múltiples sitios Web tipo Macromedia. Hoy la Web 2.0 apuesta por una interactividad más rica donde el usuario puede moverse y operar como en su ordenador. Por ello, el diseño de la interfaz Web 2.0 propone hoy una serie de experiencias de usuario de acceso desde cualquier lugar y momento, una usabilidad más sencilla, amigabilidad del entorno $y$, como no puede ser de otro modo, en un entorno que propicia la interacción social, una capacidad para crear contenidos en forma dinámica integrados a los computadores (Rodríguez, 2017).

En este sentido, las estrategias de aprendizaje son representaciones mentales que se figuran en un plan de acción elaborado de una manera reflexiva, como secuencia de acciones dirigidas a mejorar el aprendizaje, para lo cual se requiere tomar decisiones en el uso de las diversas estrategias, con el propósito de mejorar el conocimiento.
Con referencia a lo descrito, el uso de diversas estrategias de aprendizaje potencia las habilidades, destrezas en el pensamiento y la inteligencia del estudiante, son procedimientos mentales conscientes $\mathrm{e}$ intencionales que los estudiantes definen, en la cual el alumno elige y activa, de manera coordinada, los conocimientos.

Considerando lo anterior, la función de los profesores ha cambiado, pues los conocimientos avanzan a una velocidad impresionante y no es posible implementar en la universidad planes de estudio que abarquen todo el conocimiento de una. Se busca responder a la necesidad de reforzar de manera significativa los aprendizajes de todos y cada uno de los alumnos que cursen la unidad curricular sistemas operativos, de igual forma, busca que los alumnos obtengan un mejor desenvolvimiento con respecto a las herramientas Web 2.0 para lograr un alto rendimiento en el campo laboral. Por ello, es pertinente considerar las herramientas Web 2.0, especificadas en el cuadro 1 como parte de las estrategias para desarrollar aprendizajes en la Unidad Curricular.

Cuadro 1. Herramientas Web 2.0

\begin{tabular}{lll}
\multicolumn{1}{c}{ Herramienta } & \multicolumn{1}{c}{ Función } & \multicolumn{1}{c}{ Sitio Web } \\
\hline Twitter & Red social, crear micro blogs. & https://twitter.com/ \\
Google Sites & Crear sitios Web, Google Doc's & www.google.com \\
Dropbox & $\begin{array}{l}\text { Disco duro virtual, para compartir } \\
\text { información. }\end{array}$ & https://www.dropbox.com/ \\
Skydrive & $\begin{array}{l}\text { Disco duro virtual, para compartir } \\
\text { información. }\end{array}$ & http://skydrive.com \\
\hline
\end{tabular}




\begin{tabular}{lll}
\hline \multicolumn{1}{c}{ Herramienta } & \multicolumn{1}{c}{ Función } & \multicolumn{1}{c}{ Sitio Web } \\
\hline Podcast & Grabador de voz & \\
Prezzi & Crear presentaciones multimedia & http://Prezzi.com/ \\
Blogger & Crear blog para publicar contenidos & www.blogger.com \\
Wordpress & $\begin{array}{l}\text { Crear blog para publicar contenidos } \\
\text { Red social para crear grupos de }\end{array}$ & www.wordpress.com \\
Facebook & trabajo & www.facebook.com \\
\hline
\end{tabular}

Fuente: Elaboración propia.

En consideración con lo expresado, las estrategias didácticas deben estar orientadas a utilizar mecanismo que le permitan al estudiante mantener un aprendizaje permanente, proporcionando los recursos que demanda la sociedad para crear ciudadanos capaces de enfrentarse a problemas multidimensionales utilizando todos los recursos a su alcance, en este caso se propone el uso de las herramientas Web.

\section{Inteligencia emocional}

Los docentes deben estar alistados, preparados para recibir a sus alumnos, ese grupo maravilloso de dudas que quieren ser aclaradas en un espacio propicio, por lo cual la actitud es determinante, para que pueda surgir la empatía, así como emplear estrategias idóneas a los requerimientos de las unidades curriculares a aprender.

La concepción de este término no tuvo lugar hasta el momento en el que Howard Gardner propuso el modelo de inteligencias múltiples, diferenciando entre las capacidades intelectuales y las emocionales. El desarrollo de la inteligencia emocional, debe ser uno de los objetivos prioritarios a trabajar en la etapa de la educación infantil, ya que el niño inicia su escolarización con un desarrollo madurativo cargado de energía emocional, necesidad de comunicar sus sentimientos y emociones y falta de destreza en la utilización de los cauces adecuados de comunicación de dichas sensaciones y necesidades efectivas.

En relación a lo antes mencionado de acuerdo con Alvarado, et al. (2017) las competencias han irrumpido en el mundo educativo de tal manera que cualquier planteamiento, proyecto o investigación en educación trata sobre su desarrollo o como mínimo hace alusiones o referencias a cómo se están trabajando las competencias básicas que todo ciudadano debe adquirir en su proceso educativo. Dicho esto surge la interrogante ¿Qué competencias se relacionan con el uso de las TIC en la educación?

\section{Didácticas para trabajar lo emocional en el aula}

Con estas actividades el estudiante descubre la diversidad emocional, fomenta su percepción de los sentimientos propios y ajenos, observa cómo los sentimientos motivan distintos comportamientos, percibe la transición de un estado emocional a otro (del amor al odio), es consciente de la posibilidad de sentir emociones contrapuestas (sorpresa e 
ira, felicidad y tristeza) y cómo los personajes literarios o de cine resuelven sus conflictos o dilemas personales.

Seguramente con la práctica se logren trasladar estas formas de tratar y manejar las emociones a la vida cotidiana, aprendiendo a reconocer y comprender los sentimientos de los otros educandos o educadores, enfatizando con las emociones de los demás compañeros de clase, regulando el estrés y malestar, optando por resolver y hacer frente a los problemas sin recurrir a la violencia, en definitiva, enseñando a los educandos a prevenir comportamientos violentos, desajustados emocionalmente, tanto fuera como dentro del aula. A continuación se muestran algunos ejemplos considerados por Acosta (2013) a saber:

- Cualquier contenido que el docente explique debe ser vivido por el estudiante también desde la emoción, no solo como un dato o un conocimiento más.

- El uso de fotografías y escenas de la vida diaria son elementos que provocan con bastante facilidad la emergencia del mundo emocional.

- Uso del relato, el cuento, obras de literatura o poesía.

- Las obras literarias están llenas de situaciones emocionales a partir de las cuales se pueden trabajar estos contenidos.

- La propuesta de juegos de comunicación y expresión emocional.

- Las películas que permitan el análisis de las emociones de los personajes y de las que provocan en el espectador.
- Mensajes publicitarios como elementos de toma de conciencia, respecto del impacto emocional de las imágenes.

- La música, elemento fundamental que nos ayuda a mirar hacia dentro centrando la atención en los procesos internos.

- La utilización del color y de la forma.

- En general, las artes, incluida la danza, proporcionan los medios para expresar los sentimientos y las ideas de maneras creativas y hacen que cualquier experiencia de aprendizaje sea más memorable.

- La imaginación es el vehículo para el descubrimiento, es un motivador por excelencia del aprendizaje, que suscita una emoción básica: la curiosidad.

- El aprendizaje cooperativo.

\section{MÉTODO}

La metodología elegida consistió en una investigación acción que recupera la reflexión crítica sobre la práctica docente. La investigación partió del principal problema identificado, en el cual los estudiantes, pueden verse afectados por emociones distintas al momento de aprender sistemas operativos. A partir de esto se recomiendan estrategias didácticas que permitieran el conocimiento sobre que son las herramientas de la Web 2.0.

En función de los objetivos de estudio, la investigación fue descriptiva, de campo y prospectiva; según Hernández y col. (2014) quienes aseguran que la investigación de este tipo busca especificar propiedades, características y rasgos importantes de cualquier fenómeno que se analice. 
Por consiguiente, el estudio se desarrolló bajo el enfoque del paradigma positivista, orientado por las acciones de un análisis cuantitativo, sienta sus bases en la concepción filosófica, según la cual fuera de nosotros existe una realidad totalmente hecha, acabada, plenamente externa, objetiva y que los seres humanos, podemos reflejarla y representarla matemáticamente.

En relación con las ideas expresadas, el positivismo involucra la ley de los tres estadios de la evolución del pensamiento, el teológico, el metafísico y el científico o positivo, siendo este último el más avanzado, puesto que construye el saber fundamental en la experiencia y en la observación; en tal sentido, Hurtado (2010), refiere que es propio de este tipo de investigación, demostrar como la acción didáctica del docente contribuye o no, a otorgarle a la profesión docente un nuevo significado, una nueva función en el campo social $y$ profesional, de manera que el educador valore su ser y deber ser.

En relación a la población del presente estudio estuvo conformada por 1.423 estudiantes que cursan en el Programa de Humanidades y Educación, de los cuales 469 estudian la Licenciatura en Informática Educativa y 31 cursan la Unidad Curricular Sistemas Operativos de la Licenciatura en Educación Mención Informática del Núcleo LUZ COL, donde, dichas características se presentan a en el cuadro 2 .

Cuadro 2. Distribución de la población

\begin{tabular}{cc}
\hline LUZ-COL & Alumnos \\
\hline Programa de Humanidades y Educación & 1.423 \\
Licenciatura en Informática Educativa & 469 \\
Unidad curricular Sistemas Operativos & 31 \\
\hline
\end{tabular}

Fuente: Secretaría docente PHE-LUZ-COL

Para efectos del estudio y lograr tomar la información, se consideró una porción de la población, definida como la muestra, considerada por Hernández y col. (2014), y Méndez (2011), como la proporción representativa de la población, es decir, es un subgrupo de ella que permite generalizar los resultados de la investigación, la cual tiene por finalidad integrar todas las observaciones (sujetos, objetos, situaciones, instituciones, organizaciones o fenómenos). La población para este estudio es censal, es decir se tomaron los 31 estudiantes que cursan la
Unidad Curricular Sistemas Operativos.

De igual manera, para tal fin se diseñó un cuestionario, constituido por 18 preguntas cerradas, de tipo dicotómico (si o no), para medir la dimensión orientada a identificar las herramientas Web aplicadas por los estudiantes de la Unidad Curricular Sistemas Operativos de la Licenciatura en Educación, mención Informática del Núcleo LUZ-COL, esto con la intención de configurar el entorno personal de aprendizaje.

Por consiguiente, una vez diseñados los instrumentos, los mismos fueron sometidos a 
un estudio técnico para la identificación de la validez y confiabilidad. De acuerdo a lo expuesto por Hernández y col. (2014), la validez se refiere al grado en que un instrumento realmente mide la variable que pretende medir, mientras la confiabilidad se refiere al grado en que su aplicación repetida al mismo sujeto u objeto produce los mismos resultados.

Para el cálculo del coeficiente de confiabilidad, se utilizó el software estadístico SPSS, V. 24, el cual, determina esta característica a partir de diversos métodos. Para este caso, se utilizó el coeficiente Alfa de Cronbach, obteniéndose un valor de 0.951, lo cual representa un instrumento altamente confiable.

RESULTADOS

Las instituciones educativas en muchas ocasiones utilizan el aprendizaje interconectado para llevar a cabo las prácticas de aprendizaje de toda la vida, sin atender al nuevo ecosistema comunicacional y a las herramientas que permiten mejorar las experiencias de aprendizaje ligadas a los entornos comunicativos de alumnas $y$ alumnos. La convivencia y resolución de conflictos, se ven afectados por las nuevas formas de comunicación e interacción provenientes del empleo de las tecnologías, por lo que los diversos profesionales encargados del acto educativo deberían estimar su beneficio o desventajas en las aulas.

Con referencia al objetivo dirigido a especificar los tipos de herramientas de las estrategias Web 2.0 personales que utilizan los estudiantes en la unidad curricular sistemas operativos, en la Universidad del Zulia, específicamente del Programa de Educación Mención Informática del Núcleo $\mathrm{COL}$, se presentan a continuación las preferencias que tienen los estudiantes.

Tabla 1. Herramientas Web personales

\begin{tabular}{lcccc}
\hline Herramientas Web & $\mathbf{S i}$ & \% & No & \% \\
\hline Twitter & 2 & 6 & 29 & 94 \\
Google Sites & 3 & 10 & 28 & 90 \\
Dropbox & 29 & 94 & 2 & 6 \\
Skydrive & 8 & 26 & 23 & 74 \\
Podcast & 10 & 32 & 21 & 68 \\
Prezzi & 31 & 100 & 0 & 0 \\
Blogger & 7 & 23 & 24 & 77 \\
Wordpress & 30 & 97 & 1 & 3 \\
Facebook & 3 & 10 & 28 & 90 \\
\hline
\end{tabular}

Fuente: Elaboración propia. 
Se obtuvo un $100 \%$ de preferencia en el uso del Prezzi un 97\% utiliza el Wordpress, el 94\% el Dropbox, que serían las herramientas de mayor uso por los estudiantes. En este sentido, el Prezzi es una herramienta que está desplazando las presentaciones tradicionales en PowerPoint, utilizada en muchas universidades para las clases y presentación de investigaciones. Pero la mayor parte de las actividades se dan en un nivel no planeado estratégicamente.

Con respecto al trabajo con Prezzi, para crear presentaciones multimedia, algunos de los comentarios tenían que ver con la originalidad de Prezzi. También los estudiantes expresaron ventajas importantes de esta herramienta como la facilidad de publicar directamente en Internet la presentación, además de ser compatible con muchos sistemas operativos.

Por otra parte, los estudiantes ya no producen solamente para el profesor sino que su público se extiende facilitando la retroalimentación crítica como consecuencia de los comentarios de los lectores. Éstos pueden ser profesores, compañeros, padres o integrantes de la audiencia general.

En este orden de ideas, el uso de los blogs por los estudiantes tiene un amplio espectro. Un sencillo blog de autor puede usarse para proporcionar un espacio propio on line, para plantear preguntas, publicar trabajos $\mathrm{y}$ comunicarse. Herramientas como WordPress, y Dropbox, son muy interesantes para crear y respaldar información, en el espacio Web de un centro o proyecto educativo, asignatura o contenidos importantes. WordPress permite la publicación de contenidos de una forma fácil, con un sin número de aplicaciones, se pueden colocar links, información, comentarios y sistemas de evaluación, donde el estudiante puede interactuar con la información, el profesor y hasta autoevaluarse.

Asimismo los alumnos calificaron a los blogs como una forma de trabajo entre excelente y muy buena, $95 \%$; el otro $5 \%$ restante la catalogó como buena forma de trabajo. Referente al trabajo con blogs, algunos de los comentarios destacaron las cualidades didácticas de la herramienta, tanto para el profesor como para el alumno. Los estudiantes consideraron los foros como una forma de trabajo entre excelente y muy buena, 95\%; el otro $5 \%$ la clasificó simplemente como buena forma de trabajo. Las estrategias Web 2.0, pueden desarrollar la inteligencia emocional, en aspectos como el control, expresión adecuada de emociones, liderazgo, afectividad, empatía y toma de decisiones.

Por otra parte, la aplicación del instrumento de uso de la Web 2.0 permitió valorar la importancia que dan los estudiantes a determinadas herramientas, y posibilitó la identificación de su relación con la inteligencia emocional, demostrando que reduce el impacto del aprendizaje en la Unidad Curricular Sistemas Operativos.

\section{CONCLUSIONES}

Se considera que la educación es la tarea fundamental de la sociedad en su proceso de formar a las nuevas generaciones a fin de mantener, conservar y enriquecer el patrimonio cultural de una nación. La educación formal constituye un factor importante en el desarrollo de la sociedad, lo que significa despertar el sentido crítico, la innovación y el aprendizaje mediante el uso de las tecnologías de la información y la comunicación, en un contexto en permanente cambio. 
Las herramientas de la Web 2.0 son del agrado de los jóvenes universitarios, éstas sumadas a estrategias didácticas adaptadas al tipo de contenidos de las materias que se imparten, son herramientas valiosas para el profesor ya que a través de ellas el profesor puede agregar actividades dinámicas que permitan hacer llegar los conocimientos a los estudiantes de formas innovadoras. Son herramientas que permiten fomentar entre los estudiantes tanto el trabajo colaborativo como el trabajo individual.

En función de los resultados obtenidos, en función de las herramientas Web 2.0 aplicadas por los estudiantes éstas permiten que los jóvenes adquieran competencias necesarias a fin de desarrollar el pensamiento crítico, la colaboración, competencias digitales, estimula el aprendizaje y trabaja en redes, entre otras.

En este orden de ideas, los estudiantes quienes aplicaron las herramientas de la Web 2.0, mejoraron la forma de trabajo en el desarrollo de materiales académicos; comunicación y presentación de la información. Esto permitió a su vez generar contenidos de mayor calidad, al trabajar de forma individual y colaborativa, además se optimizaron la comunicación y organización en los horarios habituales de clases como fuera de clases.

Finalmente, el docente, como creador y promotor de experiencias para la producción de conocimientos que induzca el trabajo en el aula y fuera de ella, debe propiciar la participación y vinculación de todos los estudiantes de las diversas tareas cotidianas, desde plantear los problemas hasta la reflexión y búsqueda de las posibles respuestas a los mismos, mediante el uso de las herramientas Web 2.0 y el desarrollo de la inteligencia emocional.

Observación: Este trabajo de investigación forma parte del proyecto, "ESTRATEGIAS WEB 2.0 PARA EL DESARROLLO DE LA INTELIGENCIA EMOCIONAL". Registrado ante el CONDES (Consejo de Desarrollo Científico, Humanístico y Tecnológico de la Universidad del Zulia), bajo el número VAC-CONDES- $\mathrm{CH}$ 0308-17.

\section{REFERENCIAS}

Acosta J. (2013). PNL (Programación Neurolingüística) e Inteligencia Emocional. Barcelona: Amat Editorial y Profit Editorial

Alvarado, J. Martillo, I. y Torrech, J. (2017) Estrategias metodológicas activas vinculadas a las TIC: Utilización de estrategias metodológicas activas basadas en la web 2.0 para mejorar el proceso de enseñanza aprendizaje. España, Madrid: Editorial Académica Española

Goleman, D. (2013). Focus, Desarrollar la atención para alcanzar la excelencia. (traducción de David González Raga y Fernando Mora).España, Barcelona: Editorial Kairós, S.A

Hernández, R. Fernández, C. y Baptista, P. (2014). Metodología de la Investigación. México: Editorial Mc. Graw Hill

Méndez, C. (2011). Metodología: diseño y desarrollo del proceso de investigación con énfasis en ciencias empresariales. México: Editorial Limusa

Rodríguez, F. (2017). El desarrollo de las competencias básicas con aplicaciones Web 2.0, España, Madrid: Editorial La Muralla S.A.

Rodríguez, D. (2017) Aplicaciones Google. Bogotá: Ediciones de la U Ltda 